\title{
Clinical signs, treatment, and outcome for California sea lions (Zalophus californianus) with Sarcocystis-associated polyphasic rhabdomyositis
}

\author{
Sophie T. Whoriskey DVM \\ Pádraig J. Duignan DVM, PhD \\ Abby M. McClain DVM \\ Mauricio Seguel DVM, PhD \\ Frances M. D. Gulland VetMB, $\mathrm{PhD}$ \\ Shawn P. Johnson DVM, MPVM \\ Cara L. Field DVM, PhD
}

From the Department of Veterinary Medicine and Science, The Marine Mammal Center, Sausalito, CA 94965 (Whoriskey, Duignan, McClain, Gulland, Johnson, Field); and Department of Pathobiology, Ontario Veterinary College, University of Guelph, Guelph, ON NIG 2WI, Canada (Seguel).

Address correspondence to Dr. Whoriskey (whoriskeys@ tmmc.org).

\begin{abstract}
OBJECTIVE
To describe clinical signs, treatment, and outcome for California sea lions (Zalophus californianus) with Sarcocystis-associated polyphasic rhabdomyositis.
\end{abstract}

\begin{abstract}
ANIMALS
38 free-ranging juvenile to adult California sea lions examined at a rehabilitation center in California between September 2015 and December 2017.
\end{abstract}

\section{PROCEDURES}

Medical records at The Marine Mammal Center were reviewed to identify sea lions in which sarcocystosis had been diagnosed.

\section{RESULTS}

Clinical signs were highly variable and associated with polyphasic rhabdomyositis attributed to Sarcocystis neurona infection. Generalized severe muscle wasting, respiratory compromise, and regurgitation secondary to megaesophagus were the most profound clinical findings. Respiratory compromise and megaesophagus were associated with a poor prognosis. Eight of the 38 sea lions were treated and released to the wild, and 2 subsequently restranded and were euthanized. Two additional animals received no targeted treatment and were released. The remaining 28 animals were either euthanized or died during treatment.

\section{CONCLUSIONS AND CLINICAL RELEVANCE}

Results suggested that unlike other marine mammals, which typically develop encephalitis, California sea lions with sarcocystosis often have polyphasic rhabdomyositis with highly variable clinical signs and that extensive diagnostic testing may be required to confirm the diagnosis. Treatment with an antiprotozoal drug in combination with corticosteroids may resolve clinical disease, but the prognosis is guarded.
$I^{n}$ nfection with protozoal parasites in the genus Sarcocystis is a disease affecting wild and domestic taxa worldwide. In North American terrestrial mammals, Sarcocystis neurona causes some of the most severe forms of sarcocystosis, including fatal myelitis and encephalitis. ${ }^{1}$ Sarcocystis neurona has a 2-stage life cycle. The opossum (Didelphis virginiana) is the only definitive host in North America, but numerous intermediate hosts have been documented, including the armadillo (Dasypus novemcinctus), skunk (Mephitis mephitis), dog (Canis familiaris), and southern sea otter (Enhydra lutris) ${ }^{1-5}$ Sarcocystis neurona reproduces sexually in the intestinal epithelium of opossums, generating sporocysts containing 4 sporozoites. Ingestion of contaminated feces or water can result in intermediate host infection, with various effects in the intermediate host. The parasite typically forms latent sarcocysts in myocytes of striated muscle in intermediate hosts, and sarcocyst-containing muscle is the source of infection for opossums. ${ }^{6}$ In other intermediate or incidental hosts, organisms may localize to the brain, liver, or skeletal muscle, and disease pathogenesis varies with host and tissue tropism.

Sarcocystis neurona can cause severe disease in both terrestrial and marine mammals $1,7,8$ and is best known as the causative agent of equine protozoal my- eloencephalitis in horses. Disease can be acute or chronic, with rapid or slow progression and with focal or multifocal neurologic signs. Clinical signs vary, but ataxia, asymmetric weakness, cranial nerve deficits, and spasticity of all 4 limbs are common, and seizures can occur in some cases. ${ }^{1}$ As in horses, $S$ neurona is a documented cause of meningoencephalitis in free-ranging southern sea otters (Enhydra lutris nereis) and Pacific harbor seals (Phoca vitulina richardsi). ${ }^{7-11}$ Infection in these species can lead to stranding with neurologic signs, including depressed mentation, tremors, seizures, and coma. ${ }^{7,9,10}$ Owing to the severity of clinical signs, affected individuals are frequently euthanized or die shortly after stranding. In harbor seals, the cerebellum is severely impacted, ${ }^{9}$ and tremors consistent with cerebellar disease were the most important clinical finding in 1 infected animal. ${ }^{11}$ A novel genotype of $S$ neurona has been identified in pinniped and cetacean species of the Pacific Northwest and is associated with severe, frequently fatal encephalitis. ${ }^{8}$ The source of this genotype is unclear, and little is known about the epidemiology of this parasite in marine mammals.

Sarcocystosis is also a documented cause of hepatic disease and myopathies in multiple species,,12-16 including marine mammals, ${ }^{13-20}$ but these are reported 
less frequently than the neurologic manifestations associated with $S$ neurona. In addition, although Sarcocystis-associated myositis has been recognized in California sea lions (CSLs; Zalopbus californianus) for at least a decade, ${ }^{17,19,20}$ there is little published information on clinical signs and treatment strategies. ${ }^{17,20}$

Polyphasic rhabdomyositis ${ }^{19}$ associated with Sarcocystis spp was observed in stranded CSLs treated between 2015 and 2017 at a rehabilitation center in California. The purpose of the study reported here was to describe clinical signs, treatment, and outcome for CSLs with Sarcocystis-associated polyphasic rhabdomyositis.

\section{Materials and Methods}

Medical records at The Marine Mammal Center in Sausalito, California, were reviewed to identify CSLs stranded between September 1, 2015, and December 1, 2017 , in which a diagnosis of sarcocystosis was made. Cases were included in the study if any of the following criteria were met: positive results for a serologic test for anti-S neurona antibodies; histologic evidence in a muscle biopsy specimen of polyphasic lymphohistiocytic myositis, protozoal cysts consistent with Sarcocystis spp, or both; and gross or histologic findings consistent with sarcocystosis.

During the time of the study, stranded CSLs along a 600-mile stretch of the Central-Northern California coast between San Luis Obispo county and Mendocino county were admitted to The Marine Mammal Center for clinical evaluation and rehabilitation. On admission, each animal received a full physical examination. Sex was determined from an examination of the external genitalia, and body weight and length (tip of snout to tip of tail) were measured. Age class (pup, yearling, juvenile, subadult, or adult) was estimated through a combination of body length, tooth size, and, in males, stage of sagittal crest development. ${ }^{21,22}$

Blood was collected with a 1.5-inch 20-gauge needle from the caudal gluteal vein directly into evacuated tubes. All CBCs and serum biochemical analyses were conducted in-house with automated analyzers within the first 48 hours after admission. Complete blood counts were performed on blood anticoagulated with EDTA. White blood cell differential counts were performed manually by a small group of trained personnel on blood smears stained with Wright-Giemsa stain. Serum biochemical analyses included measurement of iron, total protein, albumin, globulins, glucose, bilirubin, urea nitrogen, creatinine, sodium, chloride, potassium, calcium, and phosphorus concentrations and $\gamma$-glutamyltransferase, alanine transaminase, aspartate transaminase, alkaline phosphatase, and creatine kinase activities.

Additional diagnostic tests were conducted on an individual basis, including abdominal ultrasonography, thoracic radiography, endoscopy, MRI, echocardiography, muscle biopsy, and $S$ neurona serologic testing. A full necropsy was performed on all animals that died or were euthanized, and histologic examination of tissues collected at the time of necropsy was performed on most of these animals.
For 21 animals, serum obtained at the time of admission was submitted to the University of California-Davis Clinical Diagnostic Laboratory for measurement of anti-S neurona antibody titer with an indirect fluorescent antibody test developed and validated for use with southern sea otter serum. ${ }^{23}$ An IgG titer $>1: 320$ was considered a positive result on the basis of previous reports. ${ }^{11,17,23,24}$

Abdominal ultrasonography (liver, kidneys, lower urogenital tract, and spleen) was typically conducted under manual restraint or sedation, whereas other diagnostic imaging and muscle biopsy were performed under general anesthesia. Anesthetic protocols included sedation with midazolam ( 0.2 to $0.3 \mathrm{mg} / \mathrm{kg}$, IM) and butorphanol ( 0.1 to $0.3 \mathrm{mg} / \mathrm{kg}$, IM), with the addition of medetomidine ( 0.01 to $0.03 \mathrm{mg} / \mathrm{kg}$, IM) for more fractious patients. Isoflurane delivered in oxygen via a mask was used for anesthetic induction and maintenance, and patients were generally intubated with an appropriately sized endotracheal tube. Diagnostic tests were conducted within 2 to 14 days after admission, depending on clinical signs, initial examination findings, assigned anesthetic risk, and response to initial treatments.

Muscle biopsy specimens were collected from the supraspinatus muscle over the scapula, the sternocephalicus muscle in the cervical region, or both because a previous study ${ }^{24}$ showed Sarcocystis organisms were more common in muscles cranial to and including the diaphragm. Biopsy sites were prepared with standard sterile techniques, and biopsy specimens were collected with forceps and scissors, a scalpel blade, or a 6-mm-diameter biopsy punch and were roughly 4 to 6 $\mathrm{mm}$ in diameter. Biopsy specimens were partitioned, with half of each specimen preserved in buffered $10 \%$ formalin for histologic examination and the remaining half frozen at $-80^{\circ} \mathrm{C}$ for molecular speciation. Tissues for histologic examination were processed with standard protocols and stained with H\&E stain.

Treatment protocols were based on recommendations for treatment of equine protozoal myeloencephalitis in horses ${ }^{1}$ and a few anecdotal reports on marine mammals. ${ }^{11,17,20}$ The antiprotozoal drug used was ponazuril, which was administered at a dosage of $10 \mathrm{mg} / \mathrm{kg}$, PO, every 24 hours for 28 days. Alternative coccidiostatic antimicrobials that were used were clindamycin (10 to $15 \mathrm{mg} / \mathrm{kg}$, IM or PO, q $12 \mathrm{~h}$ ) and trimethoprim-sulfonamide ( $15 \mathrm{mg} / \mathrm{kg}, \mathrm{PO}, \mathrm{q} 12 \mathrm{~h}$ ), administered for 28 days. Coccidiostatic antimicrobials were used if patients were anorectic or consistently regurgitating and ponazuril could not be administered orally, concurrent conditions existed, or only mild disease was present and an attempt was being made to assess the viability of using a more cost-effective medication in treating this disease. The use of trimethoprim-sulfonamide or clindamycin for concurrent conditions did not preclude the use of ponazuril, and some animals received a combination of all 3 drugs. To reduce inflammation associated with anticipated parasite die-off, a course of prednisone $(0.15$ to $0.5 \mathrm{mg} / \mathrm{kg}$, PO, q 12 h) was administered, with the dosage tapered 
over a 2 - to 4 -week period. In anorectic patients, dexamethasone $(0.2 \mathrm{mg} / \mathrm{kg}, \mathrm{IM}, \mathrm{q} 24 \mathrm{~h}$ and tapering to 0.1 $\mathrm{mg} / \mathrm{kg}$, IM, q $24 \mathrm{~h}$ over 3 days) was administered. For 3 animals in which sarcocystosis was suspected but concurrent disease was diagnosed, carprofen $(2 \mathrm{mg}$ / $\mathrm{kg}$, PO, q $12 \mathrm{~h}$ ) or ketoprofen ( $1 \mathrm{mg} / \mathrm{kg}$, IM, q $24 \mathrm{~h}$ ) was administered instead of corticosteroids.

Additional antimicrobial and gastroprotectant medications were administered on a case-by-case basis for treatment of concurrent diseases, including pneumonia, vomiting, regurgitation, and persistent diarrhea. For regurgitation or vomiting, ondansetron $(0.2 \mathrm{mg} / \mathrm{kg}, \mathrm{IM}, \mathrm{q} 24 \mathrm{~h})$ or maropitant citrate (1 mg/ $\mathrm{kg}, \mathrm{PO}, \mathrm{q} 24 \mathrm{~h}$ ), alone or in combination with metoclopramide (0.2 to $0.3 \mathrm{mg} / \mathrm{kg}$, IM, q $8 \mathrm{~h})$, was admin-

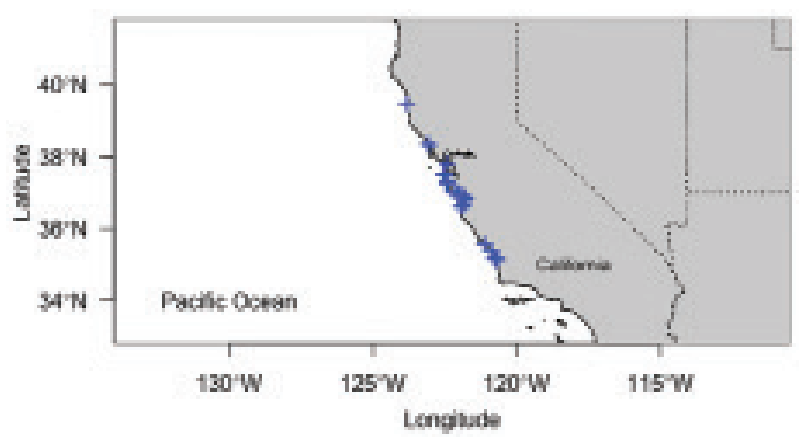

Figure I-Location of stranding for 38 Califonia sea lions (CSLs; Zalophus californianus) with Sarcocystis-associated polyphasic rhabdomyositis examined at The Marine Mammal Center in Sausalito, California, between September I, 2015, and December I, 2017.

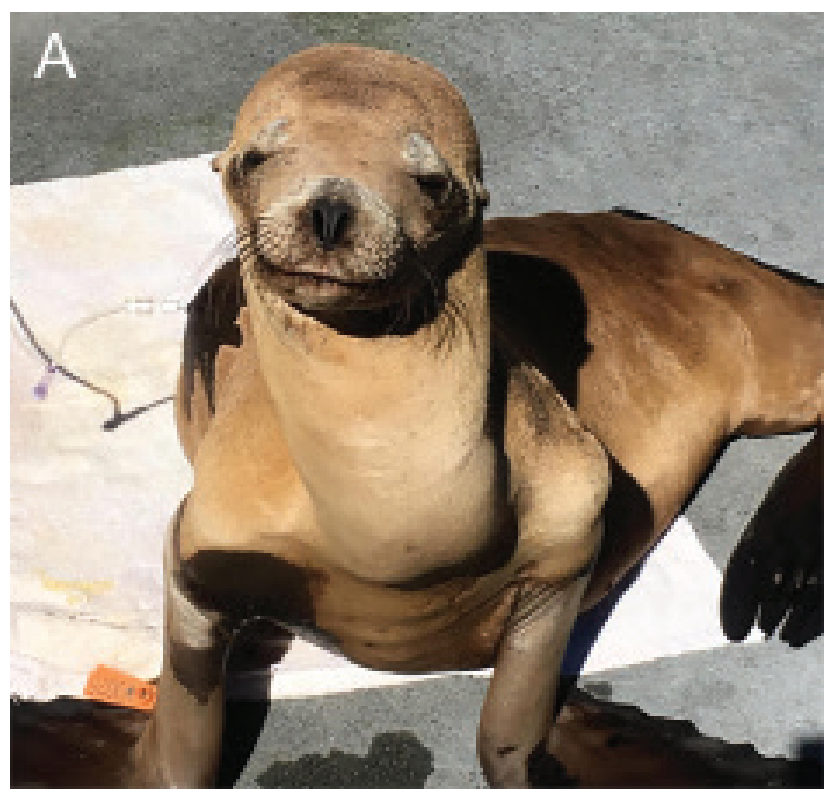

Figure 2-Photographs of 2 stranded CSLs examined at The Marine Mammal Center. A-An adult female CSL with Sarcocystis infection; notice the marked atrophy of muscle and fat. $\mathrm{B}$-An adult female CSL with signs of domoic acid toxicosis and normal body condition. The approximate locations for collection of biopsy specimens from the supraspinatus muscle over the scapula (red arrow) and the sternocephalicus muscle in the cervical region (blue arrow) are indicated.

istered. Antimicrobials used included ciprofloxacin (15 mg/kg, PO, q $24 \mathrm{~h}$ ), doxycycline (5 mg/kg, PO, q $12 \mathrm{~h})$, ceftiofur $(6.6 \mathrm{mg} / \mathrm{kg}$, IM, q $5 \mathrm{~d})$, and oxytetracycline $(20 \mathrm{mg} / \mathrm{kg}$, IM, q $3 \mathrm{~d})$. All animals received crystalloid fluids ( 20 to $30 \mathrm{~mL} / \mathrm{kg}$, SC, q $12 \mathrm{~h}$ ) during the first 48 to 72 hours of rehabilitation or longer if substantial dehydration was present and the animal tolerated the procedure.

\section{Results}

\section{Signalment and clinical signs}

A total of 38 stranded CSLs between September 1, 2015, and December 1, 2017, met the criteria for inclusion in the study. This was approximately 6 times the annual number of cases recorded during the preceding 15 years by The Marine Mammal Center. Most $(n=31)$ of the animals included in the study were examined in the summer or early fall (ie, June through September). There were 19 adults, 10 subadults, and 9 juveniles; 19 were males, and the other 19 were females. Stranding locations were concentrated in central California (Figure I).

On admission, animals were weak and emaciated and often had severe, generalized muscle atrophy. The vertebrae, scapulae, ribs, and pelvic bones were typically visible, and atrophy of the temporalis and masseter muscles was particularly pronounced, giving the animals marked cranial angularity with prominent zygomatic arches, sagittal and occipital crests, and mandibular rami (Figure 2; Table I). Twentyfive animals had clinical signs of respiratory disease, including dyspnea, coughing, and increased larynge-

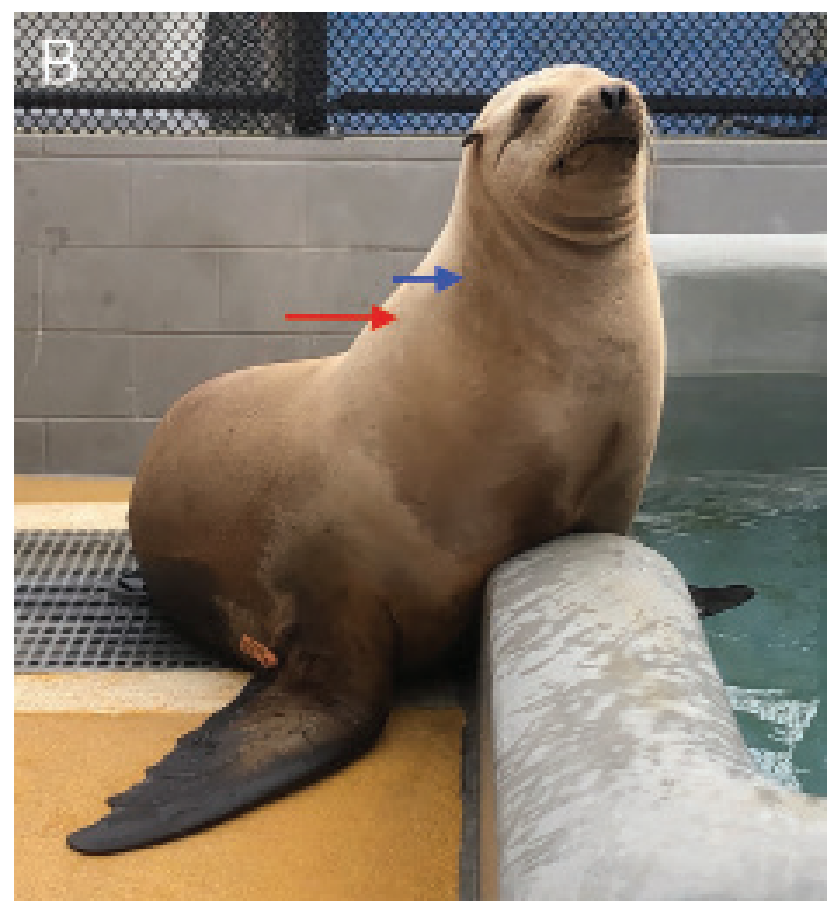


Table I-Clinical signs and treatments for 38 California sea lions (CSLs; Zalophus californianus) with Sarcocystis-associated polyphasic rhabdomyositis examined at The Marine Mammal Center in Sausalito, California, between September I, 2015, and December I, 2017.

\begin{tabular}{lcc} 
& \multicolumn{2}{c}{ No. of CSLs } \\
\cline { 2 - 3 } Variable & $\begin{array}{r}\text { Released } \\
(\mathbf{n}=\mathbf{~} \mathbf{0})\end{array}$ & $\begin{array}{c}\text { Died or } \\
\text { euthanized } \\
(\mathbf{n}=\mathbf{2 8})\end{array}$ \\
\hline Clinical signs & & \\
Diffuse muscle atrophy & 10 & 28 \\
Regurgitation, dysphagia, or hypersalivation & 2 & 10 \\
Megaesophagus & 0 & 7 \\
Respiratory signs & 6 & 19 \\
Abnormal mentation & 0 & 2 \\
Treatment & & \\
Ponazuril alone & 4 & 4 \\
Clindamycin alone & 0 & 2 \\
Ponazuril and clindamycin & 4 & 3 \\
Trimethoprim-sulfonamide & 1 & 3 \\
Corticosteroid & 7 & 6 \\
NSAID & 3 & 8
\end{tabular}

Table 2-Summary of results of diagnostic testing performed during rehabilitation for the CSLs in Table I.

\begin{tabular}{lcc} 
& \multicolumn{2}{c}{ No. of CSLs } \\
\cline { 2 - 3 } Variable & $\begin{array}{r}\text { Released } \\
(\mathbf{n}=\mathbf{~ 1 0 )}\end{array}$ & $\begin{array}{c}\text { Died or } \\
\text { euthanized } \\
(\mathbf{n}=\mathbf{2 8})\end{array}$ \\
\hline Muscle biopsy & 9 & 7 \\
Myositis & 7 & 6 \\
Follow-up muscle biopsy & 2 & 1 \\
Muscle regeneration & 2 & 1 \\
Thoracic radiography & 5 & 1 \\
Pneumonia & 4 & 8 \\
Megaesophagus & 0 & 6 \\
No specific abnormalities & 1 & 3 \\
Upper gastrointestinal tract endoscopy & 0 & 3 \\
Impaction, megaesophagus, or both & 0 & 3 \\
Abdominal ultrasonography & 9 & 16 \\
No specific abnormalities & 6 & 14 \\
Mild peritoneal fluid & 0 & 1 \\
Mild hyperechoic reniculi & 1 & 0 \\
Mild hydronephrosis & 0 & 1 \\
Mild hypoechoic reniculi & 2 & 0 \\
MRI & 0 & 2 \\
Polymyositis & 0 & 1 \\
Mild hippocampal atrophy & 0 & 1 \\
Echocardiography & 1 & 1 \\
Cardiac insufficiency & 0 & 1 \\
Mild decrease in cardiac output & 1 & 0 \\
& &
\end{tabular}

Data represent number of CSLs undergoing the procedure or number of CSLs with the specific result.

al or bronchovesicular sounds. Twelve animals had clinical signs of abnormal prehension or altered upper gastrointestinal motility, including hypersalivation, dysphagia, or persistent regurgitation of whole fish shortly after feeding. Dysphagia, regurgitation, or both were observed in 11 animals, and primary respiratory disease in combination with dysphagia, regurgitation, or both was present in 10 animals. One animal had persistent polydipsia over a period of 6 weeks but with no evidence of clinically important renal dis-

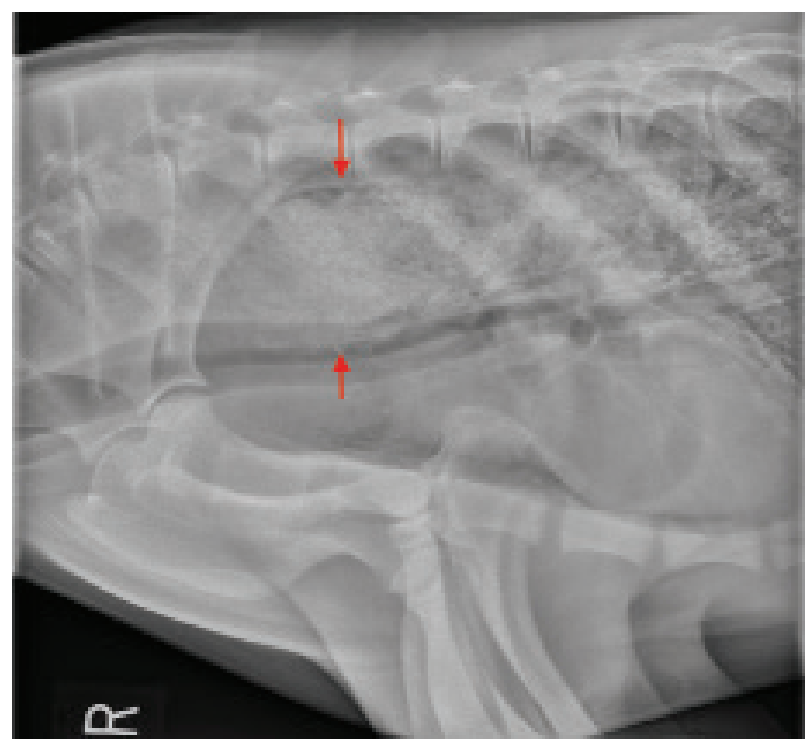

Figure 3-Right lateral thoracic radiographic image of an adult female CSL with megaesophagus secondary to Sarcocystis infection. Arrows indicate the extent of distension of the esophageal lumen. Impaction with food material is evident.

ease. One animal aborted; however, the placenta was not evaluated, so it was uncertain whether abortion was associated with sarcocystosis. For all animals, differential diagnoses included common causes of CSL stranding for these age classes, including domoic acid toxicosis, neoplasia, infectious disease (eg, sarcocystosis, toxoplasmosis, coccidioidomycosis, and leptospirosis), helminth parasitism, and malnutrition associated with environmental conditions.

\section{Diagnostic testing}

Abdominal ultrasonography was performed in 25 individuals, and in most, results were largely unremarkable (Table 2). Thoracic and cervical radiography was performed in 16 animals; 12 animals had abnormalities consistent with pneumonia, including a bronchointerstitial pattern $(n=8)$, interstitial pattern (3), and alveolar pattern (3). Nine animals suspected to have respiratory disease died or were euthanized before radiographs were obtained. Megaesophagus was diagnosed in 7 animals by means of antemortem thoracic radiography ( $=6$; Figure 3), endoscopy (3), or necropsy (1). Megaesophagus and pneumonia were both present on thoracic radiographs for 5 animals.

Endoscopy of the esophagus and stomach was performed in 3 patients with chronic regurgitation and revealed distension of the esophageal lumen and impaction with partially digested fish, supporting a diagnosis of megaesophagus. Magnetic resonance imaging was performed on 2 animals with abnormal mentation that were suspected to potentially have concurrent brain damage secondary to domoic acid exposure. Very mild hippocampal atrophy was observed in 1 patient, consistent with previous domoic acid exposure. ${ }^{25}$ Echocardiography was performed 
on 2 patients with a grade $2 / 6$ or $3 / 6$ heart murmur on presentation. Cardiac insufficiency and markedly dilated ventricles were observed in 1 individual, which was euthanized because of a poor prognosis. A mild decrease in cardiac output was detected with left ventricular muscle flattening in the second individual; these findings were considered unlikely to impact survival, and this animal was ultimately released.

Muscle biopsy was performed in 13 animals, and histologic examination of biopsy specimens consistently revealed lymphoplasmacytic and histiocytic myositis with concurrent muscle necrosis and regeneration. Intact 40- to 60- $\mu \mathrm{m}$-diameter Sarcocystis-like cysts were often present in myocytes. In 3 animals, follow-up muscle biopsy was performed 2 to 4 weeks after the initial biopsy to evaluate response to treatment. Muscle regeneration and a reduction in the severity of myonecrosis and inflammatory infiltrates were observed in all follow-up biopsy specimens. Biopsy sites healed without infection or other complications in all animals.

A CBC and serum biochemical analyses were performed on 32 animals (Table 3). Two animals had a high WBC count characterized by neutrophilia. In one of these animals, the cause of the high WBC count was unclear, and the animal was treated with a single parenteral dose of ceftiofur followed by a course of ciprofloxacin, and the WBC count normalized. The other animal with a high WBC count had concurrent advanced amyloidosis and circumferential cervical entanglement with monofilament line. Three animals had mild leukopenia. In 1, the leukopenia was characterized by neutropenia, a left shift, monocytopenia, and eosinopenia. In the remaining 2 , the leukopenia was characterized by neutropenia, eosinopenia, and, in 1 of the 2 , monocytopenia. Relative lymphocytosis (differential lymphocyte count $>$ $30 \%)$ was noted in 7 (22\%) of the 32 animals.

Serum alanine transaminase activity was high in 22 (69\%) of the 32 animals, and serum creatine kinase activity was high in 16 (50\%). Hyperphosphatemia was present in 16 (50\%), BUN concentration was high in 12 (38\%), and serum aspartate transaminase activity was high in 6 (19\%). Serum alkaline phosphatase activity, $\gamma$-glutamyltransferase activity, and creatinine concentration were each high in < $3(9 \%)$ animals. Hyperproteinemia was present in 6 (19\%) animals, and hypoproteinemia was present in 5 (16\%). Hypoalbuminemia was present in 7 (22\%) animals. Hyperglobulinemia was present in 11 (34\%) animals, and hypoglobulinemia was present in 7 (22\%). Mild hypokalemia was observed in 9 (28\%) animals, and mild hyperkalemia in 1 (3\%). Mild to severe hypernatremia was observed in 14 (44\%) animals, and mild hyperchloremia was observed in 3 (9\%). Mild hypocalcemia was observed in 4 (13\%) animals, and mild hypercalcemia was observed in 1 (3\%). For all 21 animals for which testing was performed, results of serum testing for anti-S neurona antibodies were positive (ie, serum IgG titer $>1: 320$ ).

\section{Treatment and outcome}

Ten of the 38 animals were released following rehabilitation; for these animals, mean rehabilitation time was 8 weeks (range, 4 to 14 weeks). Two of the released animals subsequently restranded. Twentythree animals were euthanized, and 5 died while being treated; 11 of these 28 animals died or were euthanized within the first 3 days of rehabilitation. The remaining 17 were in rehabilitation between 4 days and 23.5 weeks. All 7 animals in which megaesophagus was diagnosed died or were euthanized; none responded to treatment.

Eight of the 10 released animals received antiprotozoal treatment and supportive care (SC fluid administration and nutritional support), and 2 received only supportive care. Four of the released animals had concurrent pneumonia that was successfully treated with antimicrobials.

A total of 15 animals were treated with ponazuril (10 mg/kg, PO, q $24 \mathrm{~h}$ ) because they were eating. Clindamycin (10 to $15 \mathrm{mg} / \mathrm{kg}, \mathrm{IM}$ or PO, q $12 \mathrm{~h}$ ) was administered to 9 animals to treat sarcocystosis or concurrent conditions. In 7 of these 9 animals, both clindamycin and ponazuril were administered. Trimethoprim-sulfonamide ( $15 \mathrm{mg} / \mathrm{kg}, \mathrm{PO}, \mathrm{q} 12 \mathrm{~h}$ ) was administered to 4 animals to treat sarcocystosis or concurrent diseases. Three animals received both ponazuril and trimethoprim-sulfonamide, and 1 received ponazuril, trimethoprim-sulfonamide, and

Table 3-Summary of results of clinicopathologic testing performed at the time of admission for the CSLs in Table I.

\begin{tabular}{|c|c|c|c|}
\hline Variable & Reference range & Released $(n=10) *$ & Died or euthanized $(n=22)^{*}$ \\
\hline Anti-Sarcocystis neurona antibody titer & $\leq \mathrm{I}: 320$ & $\mathrm{I}: 5,120(\mathrm{I}: \mathrm{I}, 280-\mathrm{I}: 81,920)$ & $\mathrm{I}: 2,560(\mathrm{I}: 640-\mathrm{I}: 20,480) \dagger$ \\
\hline Creatine kinase $(\mathrm{U} / \mathrm{L})$ & $242-438$ & $1,007(249-4,027)$ & $1,242(209-160,400)$ \\
\hline Aspartate transaminase (U/L) & $35-69$ & $62(13-138)$ & $44(2-615)$ \\
\hline Alanine transaminase $(\mathrm{U} / \mathrm{L})$ & $25-57$ & $122(29-566)$ & $147.5(|4-|, \mid 72)$ \\
\hline Phosphorus (mg/dL) & $4.3-6.7$ & $6.7(5.3-8.9)$ & $6.75(4.5-19.1)$ \\
\hline Sodium (mmol/L) & $144-154$ & I54.I (I46.4-I68.I) & I 52.5 (I39.6-184.5) \\
\hline Potassium (mmol/L) & $4.1-5.1$ & $4.17(3.46-4.49)$ & $4.3(3.54-5.35)$ \\
\hline Globulins (g/dL) & $4.6-5.5$ & $5.4(4.5-6.9)$ & $5.05(3.5-6.4)$ \\
\hline WBC count (cells/mL) & I I,200-29,600 & $15,150(5,000-26,000)$ & $\mid 2,950(4,400-4 \mid, 000)$ \\
\hline Lymphocyte count (cells/mL) & $>30 \%$ & $2,630(1,100-4,329)$ & $2,836(628-5,882)$ \\
\hline
\end{tabular}

*Data are given as median (range). †Results were available for only II animals. 
clindamycin throughout the rehabilitation period because of the presence of multiple diseases.

The 2 animals that were released after receiving only supportive care had rapid clinical improvement before the diagnosis of sarcocystosis was made. After the diagnosis was made, these 2 animals were monitored for several weeks, but because they continued to improve clinically, specific antiprotozoal treatment was deemed unnecessary. One of these animals received ciprofloxacin for 4 weeks for treatment of pneumonia and a 3-day course of an NSAID. In the other animal, histologic examination of recheck muscle biopsy specimens collected 3 weeks after initial biopsy specimens were obtained showed muscle regeneration and repair.

Two subadult males had progressive sarcocystosis despite prolonged care (4.5 and 6.75 weeks) and multiple treatments. Both initially had generalized muscle wasting but were alert and active and eating well. Both were treated with trimethoprim-sulfonamide PO after serologic detection of anti-S neurona antibodies on admission, and both developed acute anorexia following an anesthetic procedure. One developed vomiting and regurgitation; the other became progressively dyspneic over a week. Creatine kinase activities increased rapidly in both animals, with peak activities of 67,010 and 98,960 U/L, and in 1 animal, the anti-S neurona antibody titer increased from $1: 2,560$ to $1: 40,960$ six weeks later. In 1 animal, azotemia (BUN, $63 \mathrm{mg} / \mathrm{dL}$; creatinine, $1.1 \mathrm{mg} / \mathrm{dL}$ ) and myoglobinuria developed with large volumes of darkbrown urine observed and a $4+$ heme reaction on urinalysis. Both animals were ultimately euthanized because of a progressive decline in their condition and a lack of response to treatment.

Two animals that were released after treatment stranded again, one 7.25 weeks and the other 12 weeks after release. The animal that restranded after 12 weeks was emaciated and severely debilitated and was euthanized. Histologic examination revealed mild rhabdomyositis and chronic cystitis with ascending infection, which was considered the primary disease process. The other restranded animal received a second course of treatment, and physical therapy was initiated because of an abnormal gait characterized by primarily inching forward on its chest and abdomen rather than walking upright with its pectoral flippers. Despite evidence of myocyte regeneration in serial biopsy specimens collected over a period of 18.5 weeks while the animal was under care and continued improvement in gait, the patient died while anesthetized for a medical examination. At necropsy, there was massive atrophy of the pectoral muscles, the primary locomotor muscles for otariid pinnipeds, despite prolonged care and repeated antiprotozoal treatment.

\section{Histologic findings}

Histologic examinations were performed on tissues from 23 of the deceased animals, and lesions were consistent with those previously described for polyphasic rhabdomyositis in CSLs. ${ }^{19}$ Mild meningoencephalitis consistent with sarcocystosis was observed in 5 animals, and meningitis was observed in 2 . Immunohistochemical staining to identify protozoa in the brain was not performed. Hippocampal atrophy consistent with domoic acid toxicosis was observed in 3 animals, and in 2 of these animals, meningitis or meningoencephalitis was present concurrently. Mild myocarditis associated with sarcocystosis was observed in 3 animals.

In 1 animal, disseminated urogenital carcinoma was present in addition to sarcocystosis. In this animal, neoplasia was considered the main cause of stranding, and sarcocystosis was considered a secondary disease. Myoglobinuric nephrosis was diagnosed in 5 of 9 animals with renal lesions, whereas 3 animals had interstitial nephritis suggestive of leptospirosis. The remaining animal with nephritis had evidence of an ascending bacterial infection secondary to concurrent cystitis. Gastric or duodenal ulcers or both were present in 14 animals.

\section{Discussion}

Sarcocystosis has been described in stranded marine mammals throughout the Pacific Northwest, typically presenting as CNS disease. In contrast, in the CSLs discussed in the present report, this disease manifested primarily as severe systemic muscular disease. A wide range of clinical signs were observed, generally associated with the various muscle groups that were affected. Interestingly, meningoencephalitis was noted on histologic examination of several animals and likely developed secondary to protozoal disease; however, clinical signs were consistent with musculoskeletal disease in those animals and not specific for neurologic disease. Treatment was effective in some animals; however, most animals had advanced clinical signs when first examined, and antiprotozoal medications were ineffective in reversing the disease process.

Gross and histopathologic changes associated with sarcocystosis in stranded CSLs have been previously described ${ }^{24}$; however, the present case series demonstrated the highly variable and often diagnostically challenging clinical manifestations of this disease. In addition to advanced atrophy of skeletal muscle, clinical signs were often suggestive of respiratory or gastrointestinal disease because of selective myositis of striated muscles associated with breathing (eg, the diaphragm and intercostal, pectoral, and laryngeal muscles) or swallowing (eg, esophageal muscles). Dysphagia has been described in horses with sarcocystosis and is attributed to inflammation of the nerves. ${ }^{1}$ In the cases described in the present report, dysphagia appeared to be secondary to pronounced inflammation, necrosis, and atrophy of the laryngeal, esophageal, and masticatory muscles. ${ }^{19}$

Clinically important respiratory compromise was regularly observed in the animals described in the present report. Concurrent verminous and bacterial 
bronchopneumonia has been documented previously in CSLs with rhabdomyositis. ${ }^{19}$ Compromise of the diaphragm and intercostal muscles likely reduced the ability to cough, contributing to the accumulation of exudate or development of aspiration pneumonia in some cases. Dysphagia and megaesophagus also likely increased the risk of aspiration. Regurgitation and megaesophagus have been reported once in association with Sarcocystis arieticanis infection in a $\operatorname{ram}^{26}$ and appear to be rare sequelae of this disease. In the present report, difficulty swallowing or an inability to swallow properly because of megaesophagus was associated with a grave prognosis.

In CSLs, the most specific circulating skeletal muscle enzyme is creatine kinase, ${ }^{27}$ and CSLs also have high alanine transaminase activities in cardiac muscle, followed by the liver, skeletal muscle, and the kidneys. High activities of aspartate transaminase are found in cardiac muscle, the liver, skeletal muscle, and a number of other organs and appear to have relatively low organ specificity. ${ }^{27}$ In the present report, creatine kinase and alanine transaminase activities were the most consistently high clinicopathologic variables on admission. Given the extensive skeletal muscle disease noted in these animals, even higher creatine kinase activities might have been expected, as documented previously in a captive CSL treated for this disease. ${ }^{20}$ Progression of disease and concurrent increases in creatine kinase activity were observed in 2 CSLs in the present case series; however, a lack of high creatine kinase activity did not preclude severe sarcocystosis-associated polymyositis. Creatine kinase is a highly labile enzyme, and the chronicity of disease likely accounted for the normal creatine kinase activities in many of these animals. Two dogs and a CSL with severe myositis due to sarcocystosis had high alanine transaminase activity and severe thrombocytopenia. ${ }^{12,20}$ Sarcocystis canis-induced hepatitis has been described in multiple marine mammal species, including CSLs, ${ }^{13-16,18}$ but serum biochemical values were not reported because the diagnosis was made on histologic examination. In the present case series, hepatic disease associated with sarcocystosis was minimal and, thus, unlikely to have contributed to increases in alanine transaminase activity. At least 3 individuals had histologic evidence of myocarditis, suggesting that increases in alanine transaminase activity were more likely to have arisen because of cardiac or skeletal muscle damage.

In the present case series, several electrolyte abnormalities were noted on clinicopathologic testing at the time of admission, most notably hyperphosphatemia, hypernatremia, and hypokalemia. High BUN concentrations were also present in a substantial number of cases. In the authors' experience, high BUN concentrations and electrolyte abnormalities are common in stranded CSLs owing to various degrees of dehydration and malnutrition on admission, and in these cases did not appear to be disease specific. The most severe increases in phosphorus $(19.1 \mathrm{mg} / \mathrm{dL})$, sodium $(184.5 \mathrm{mg} / \mathrm{dL})$, and BUN $(366 \mathrm{mg} / \mathrm{dL})$ concentrations occurred in an animal with concurrent renal amyloidosis. The high number of concurrent gastric and duodenal ulcers may also have contributed to high BUN concentrations. Inflammatory indicators, including WBC count and globulins concentration, were not consistently high. Interestingly, relative lymphocytosis was present in most animals, but because total WBC count was not consistently high, the clinical importance of this relative lymphocytosis was unknown. Still, relative lymphocytosis warrants further investigation, given the apparent role of lymphocytes in the inflammatory process of this disease ${ }^{19}$ Changes in other serum biochemical values were inconsistent in these cases.

Serologic testing and histologic examination of muscle biopsy specimens were the most useful methods for the diagnosis of active infection in the present case series, and repeated muscle biopsy in conjunction with clinical progress were most useful in evaluating the response to treatment. The serologic test that was used has not been validated for CSLs but has been previously used in CSLs and other marine mammals, ${ }^{11,17,20,21,24,28}$ and the results support its efficacy in these species. In a previous report ${ }^{19}$ DNA sequencing of muscle tissue from 10 CSLs with rhabdomyolysis and a positive serologic test result showed a $97 \%$ to $99 \%$ identity to $S$ neurona, with higher titers associated with more severe myonecrosis. An increase in serologic titer with progression of disease was also documented in the present case series; however, admission titer and subsequent changes did not consistently reflect the extent of disease. A retrospective seroprevalence study ${ }^{24}$ found that $2 \%$ to $13 \%$ of stranded CSLs over a 10-year period had positive serologic test results for anti-S neurona antibodies. Repeated serologic testing has been used to monitor response to treatment in captive animals ${ }^{11,20,28}$ but is a less practical monitoring tool in a rehabilitation setting.

Rhabdomyositis and Sarcocystis organisms were consistently identified in muscle biopsy specimens obtained from either collection site in the present case series. The authors preferred the supraspinatus muscle for biopsy owing to the palpable scapular landmarks and shallower depth required for access. Biopsy samples were small, however, and may not have adequately represented the extent of disease given the widely disseminated skeletal muscle involvement. Despite myocyte regeneration and reduced inflammation noted in follow-up biopsy specimens, full resolution of extensive disease was unlikely to have occurred in some or most animals while in rehabilitation. Also, an animal that remained weak and eventually died had marked atrophy of the pectoral muscles necessary for normal locomotion at necropsy, even though repeated biopsy of the sternocephalicus muscles revealed myofiber regeneration. In the authors' experience, no single diagnostic test was fully effective in the diagnosis of sarcocystosis or evaluation of the response to treatment.

The antiprotozoal drug ponazuril is a coccidiostatic benzene-acetonitrile compound that inhibits apicoplast and mitochondrial function. ${ }^{1}$ It is widely 
used in the treatment of equine protozoal myeloencephalitis and has been previously used to treat $S$ neurona infection in 3 pinnipeds. ${ }^{11,17,20}$ A captive harbor seal with $S$ neurona-associated encephalitis was treated for 3 months with resolution of clinical signs. ${ }^{11}$ A captive CSL was treated for and recovered from $S$ neurona-associated rhabdomyolysis and secondary acute renal failure and hypercalcemia. ${ }^{20} \mathrm{~A}$ stranded CSL with $S$ neurona-associated polymyositis was treated for 30 days and released, ${ }^{17}$ although the long-term outcome for this animal, and similarly for most of the released animals in the present report, was unknown. Relapses can occur in horses, and it is unknown whether treatment for $>28$ days would decrease the incidence of relapse. ${ }^{1}$ Recrudescence of disease was noted in 2 animals in the present case series that restranded, although in one of these animals, sarcocystosis was mild and the patient's primary disease on second admission was chronic cystitis and bacterial infection. However, in the other animal, sarcocystosis was the primary cause of restranding and prolonged treatment did not resolve the condition. It is unclear whether a longer duration of treatment initially would have been more effective. Interestingly, 2 animals in the present case series recovered without antiprotozoal treatment, with muscle repair noted on follow-up muscle biopsy in one of these. These findings suggested that nutritional support and rest may be sufficient to allow for recovery in some instances, although long-term follow-up was not possible for any of the released animals.

Two alternative coccidiostatic medications were used in the present case series: clindamycin and trimethoprim-sulfonamide. The lincosamide antibiotic clindamycin inhibits protein synthesis, but the precise mechanism of action against protozoa is unknown. Trimethoprim-sulfonamide inhibits folic acid synthesis in both bacteria and protozoa. In 2 animals, trimethoprim-sulfonamide alone was used as the initial primary treatment because disease signs were relatively mild at the time of admission. However, clinical disease progressed in both animals, despite treatment with this medication. Clindamycin was used in animals in which oral administration of medications was not possible, and treatment with clindamycin alone was also not successful. Animals in which clindamycin was parenterally administered had severe clinical signs associated with advanced disease, which likely contributed to the lack of success with this treatment. Although the number of patients treated with these antimicrobials was low, it seems unlikely that either will achieve therapeutic success as a single treatment.

Combinations of these drugs as well as alternative antiprotozoal drugs warrant further exploration for treatment of sarcocystosis in CSLs. Clindamycin administered with sulfadoxine-pyrimethamine (500 $\mathrm{mg}: 25 \mathrm{mg}$ ) was used in a human patient with muscular sarcocystosis, and improvement was observed within 3 days. ${ }^{29}$ Decoquinate, a 4-hydroxyquinolone coccidiostat, was used to treat a dog with $S$ neurona myositis. ${ }^{12}$ Albendazole has also been used to treat human patients during an outbreak of muscular sarcocystosis, although it was unclear whether improvements reflected successful medical treatment or the natural course of the infection. ${ }^{30}$ An in vitro study ${ }^{31}$ that examined the efficacy of pyrimethamine, trimethoprim, sulfonamides, and combinations of pyrimethamine and sulfonamides against $S$ neurona showed that pyrimethamine and trimethoprim were coccidiocidal, but sulfonamides alone were ineffective. Antiprotozoal medications, however, have unknown efficacy once sarcocysts have formed, ${ }^{31}$ and additional studies are needed to assess the viability of various medications in treating CSLs.

Although Sarcocystis cysts may be found in CSL myocytes without accompanying inflammation, ${ }^{19}$ parasite genotype is associated with disease severity and mortality rate in marine mammals. ${ }^{8}$ A marked inflammatory response has been described in CSLs with clinical sarcocystosis that is characterized by T-lymphocyte activation and subsequent infiltration of myofibers with lymphocytes, plasma cells, and macrophages rather than granulocytes. ${ }^{19}$ These findings are similar to those for immune-mediated polymyositis in humans and dogs and suggest that immune system activation plays an important role in the pathogenesis of sarcocystosis in CSLs. In humans with muscular sarcocystosis, patients given corticosteroids orally report rapid improvements in clinical signs. ${ }^{30}$ These findings suggest anti-inflammatory medications may play an important role in the treatment of this disease. Although a substantial proportion of animals in the present case series received corticosteroids or NSAIDs, the immune system response in CSLs to these medications has not been investigated and the role of the immune system in CSLs with sarcocystosis remains to be elucidated.

Similar to southern sea otters and Pacific harbor seals, which are also susceptible to Sarcocystis infection, ${ }^{32,33}$ CSLs inhabit coastal waters and are thus exposed to terrestrial protozoa. Sarcocystis sporocysts are capable of surviving in fresh water for months ${ }^{34}$ and are resistant to many commonly used disinfectants. ${ }^{35}$ Their persistence in salt water has not been studied, but the closely related apicomplexan Toxoplasma gondii has been shown to survive for up to 3 months in salt water while maintaining infectivity. ${ }^{36}$ Development of sarcocystosis in sea otters has been linked to increased rainwater runoff 1 to 2 months prior to the development of clinical signs, ${ }^{32}$ and the environmental load of coccidian parasites has been described as an underlying mechanism driving temporal changes in protozoal infections of multiple marine mammal species. ${ }^{37}$ Given the wide range of marine mammal species, including cetaceans, documented with this pathogen, $, 8,33,37$ infection most likely occurs through ingestion of infected prey or contaminated water.

The annual number of stranded CSLs with clinical sarcocystosis in the present study was approximately 6 times the annual numbers seen by The Ma- 
rine Mammal Center in the preceding 15 years. Widespread environmental shifts, including increases in sea surface temperature and reductions in prey availability for high-trophic-level species, ${ }^{38}$ led to an unusual number of deaths of CSLs in 2013 through 2017, with unprecedented numbers of stranded emaciated CSL pups and yearlings throughout the California coast. Serum IgG and IgA concentrations in CSL pups during these years were lower than concentrations in pups born during normal years, ${ }^{39}$ suggesting reduced immunocompetence. Although an increase in juvenile and adult CSL strandings was not simultaneously observed, environmental pressures may have led to immunosuppression of some individuals, predisposing them to infection or exacerbating what may have typically been a benign infection. It is also possible that the low prevalence of disease in the years prior to 2016 was related to environmental factors. From late 2011 through March 2017, California was subjected to the most intense drought in the state's history. ${ }^{40}$ Reduced rainfall and runoff may have anomalously resulted in less contamination of coastal waters by Sarcocystis sporocysts than has occurred since the resumption of more normal rainfall patterns.

In conclusion, unlike other marine mammal species described to date, stranded CSLs with sarcocystosis primarily had polyphasic rhabdomyositis rather than meningoencephalitis. Clinical signs were highly variable, with animals commonly manifesting generalized muscle wasting and respiratory compromise. Megaesophagus was associated with a grave prognosis. The diagnosis was strongly supported through a combination of serologic testing and histologic examination. Treatment success was variable and appeared to be associated with severity of disease. Given the varied presentation of this disease and its potential for recrudescence, the authors recommend multimodal treatment when possible for a minimum of 6 to 8 weeks. A course of corticosteroids is also recommended owing to the suspected immune-mediated component of this disease. The long-term prognosis for rehabilitated CSLs remains to be elucidated.

\section{Acknowledgments}

All work was conducted under National Marine Fisheries Service permit \#18786. All animal care and treatment decisions were made in accordance with animal welfare principles described by the AVMA.

\section{References}

1. Dubey JP, Howe DK, Furr M, et al. An update on Sarcocystis neurona infections in animals and equine protozoal myeloencephalitis (EPM). Vet Parasitol. 2015;209(1-2):1-42.

2. Cheadle MA, Tanhauser SM, Dame JB, et al. The nine-banded armadillo (Dasypus novemcinctus) is an intermediate host for Sarcocystis neurona. Int J Parasitol. 2001;31(4):330335.

3. Cheadle MA, Yowell CA, Sellon DC, et al. The striped skunk (Mephitis mephitis) is an intermediate host for Sarcocystis neurona. Int J Parasitol. 2001;31(8):843-849.

4. Dubey JR, Rosypal AC, Rosenthal BM, et al. Sarcocystis neurona infections in sea otter (Enhydra lutris): evidence for natural infections with sarcocysts and transmission of infection to opossums (Didelphis virginiana). J Parasitol. 2001;87(6):1387-1393.

5. Dubey JP, Sykes JE, Shelton GD, et al. Sarcocystis caninum and Sarcocystis svanai $n$. spp. (Apicomplexa: Sarcocystidae) associated with severe myositis and hepatitis in the domestic dog (Canis familiaris). J Eukaryot Microbiol. 2015;62(3):307-317.

6. Reed SM, Furr M, Howe DK, et al. Equine protozoal myeloencephalitis: an updated consensus statement with a focus on parasite biology, diagnosis, treatment, and prevention. $J$ Vet Intern Med. 2016;30(2):491-502.

7. Miller MA, Sverlow K, Crosbie PR, et al. Isolation and characterization of two parasitic protozoa from a Pacific harbor seal (Phoca vitulina richardsi) with meningoencephalomyelitis. J Parasitol. 2001;87(4):816-822.

8. Barbosa L, Johnson CK, Lambourn DM, et al. A novel Sarcocystis neurona genotype XIII is associated with severe encephalitis in an unexpectedly broad range of marine mammals from the northeastern Pacific Ocean. Int J Parasitol. 2015;45(9-10):595-603.

9. Lapointe JM, Duignan PJ, Marsh AE, et al. Meningoencephalitis due to a Sarcocystis neurona-like protozoan in Pacific harbor seals (Phoca vitulina richardsi). J Parasitol. 1998;84(6):1184-1189.

10. Miller MA, Conrad PA, Harris M, et al. A protozoal-associated epizootic impacting marine wildlife: mass-mortality of southern sea otters (Enhydra lutris nereis) due to Sarcocystis neurona infection. Vet Parasitol. 2010;172(3-4):183-194.

11. Mylniczenko ND, Kearns KS, Melli AC. Diagnosis and treatment of Sarcocystis neurona in a captive harbor seal (Phoca vitulina). J Zoo Wildl Med. 2008;39(2):228-235.

12. Sykes JE, Dubey JP, Lindsay LL, et al. Severe myositis associated with Sarcocystis spp infection in 2 dogs. J Vet Intern Med. 2011;25(6):1277-1283.

13. Garner HM, Barr BC, Packham AE, et al. Fatal hepatic sarcocystosis in two polar bears (Ursus maritimus). J Parasitol. 1997;83(3):523-526.

14. Resendes AR, Juan-Sallés C, Almeria S, Majó N, Domingo M, Dubey JP. Hepatic sarcocystosis in a striped dolphin (Stenella coeruleoalba) from the Spanish Mediterranean coast. J Parasitol. 2002;88(1):206-209.

15. Mense MG, Dubey JP, Homer BL. Acute hepatic necrosis associated with a Sarcocystis-like protozoa in a sea lion (Zalophus californianus). J Vet Diagn Invest. 1992;4(4):486-490.

16. Yantis D, Moeller R, Braun R, Gardiner CH, Aguirre A, Dubey JP. Hepatitis associated with a Sarcocystis canis-like protozoan in a Hawaiian monk seal (Monachus schauinslandi). J Parasitol. 2003;89(6):1258-1260.

17. Carlson-Bremer DP, Gulland FM, Johnson CK, Colegrove KM, Van Bonn WG. Diagnosis and treatment of Sarcocystis neurona-induced myositis in a free-ranging California sea lion. J Am Vet Med Assoc. 2012;240(3):324-328.

18. Miller M, Shapiro K, Murray MJ, et al. Protozoan parasites of marine mammals. In: Gulland FMD, Dierauf LA, Whitman KL, eds. CRC Handbook of Marine Mammal Medicine. 3rd ed. CRC Press; 2018:429-465.

19. Seguel M, Colegrove KM, Field C, Whoriskey S, Norris T, Duignan P. Polyphasic rhabdomyositis in California sea lions (Zalophus californianus): pathology and potential causes. Vet Patbol. 2019;56(4):619-629.

20. Alexander AB, Hanley CS, Duncan MC, Ulmer K, Padilla LR. Management of acute renal failure with delayed hypercalcemia secondary to Sarcocystis neurona-induced myositis and rhabdomyolysis in a California sea lion (Zalophus californianus). J Zoo Wildl Med. 2015;46(3):652-656.

21. Greig DJ, Gulland FMD, Kreuder Johnson C. A decade of live California sea lion (Zalophus californianus) strandings along the central California coast: causes and trends, 19912000. Aquat Mamm. 2005;31(1):11-22.

22. Evans K, Robertson K. A note on the preparation of sperm whale (Physeter macrocephalus) teeth for age determination. J Cetacean Res Manag. 2001;3(1):101-107.

23. Miller MA, Gardner IA, Packham A, et al. Evaluation of an in- 
direct fluorescent antibody test (IFAT) for demonstration of antibodies to Toxoplasma gondii in the sea otter (Enhydra lutris). J Parasitol. 2002;88(3):594-599.

24. Carlson-Bremer D. Epidemiology of coccidian parasites in California sea lions (Zalophus californianus). PhD dissertation. University of California-Davis; 2011.

25. Silvagni PA, Lowenstine LJ, Spraker T, Lipscomb TP, Gulland FM. Pathology of domoic acid toxicity in California sea lions (Zalophus californianus). Vet Pathol. 2005;42(2):184-191.

26. Braun U, Steiger R, Flückiger M, Bearth G, Guscetti F. Regurgitation due to megaesophagus in a ram. Can Vet J. 1990;31(5):391-392.

27. Fauquier DA, Mazet JA, Gulland FM, Spraker TR, Christopher MM. Distribution of tissue enzymes in three species of pinnipeds. J Zoo Wildl Med. 2008;39(1):1-5.

28. Fravel VA, Procter D, Field C, Colegrove K, Dover S. Sarcocys tis neurona infection in a Pacific walrus (Odobendus rosmarus divergens) and a bottlenose dolphin (Tursiops truncatus) under human care: case comparison. In: Proceedings of the 48th Annual Meeting of the International Association of Aquatic Animal Medicine 2017.

29. Italiano CM, Wong KT, AbuBakar S, et al. Sarcocystis nesbitti causes acute, relapsing febrile myositis with a high attack rate: description of a large outbreak of muscular sarcocystosis in Pangkor Island, Malaysia, 2012. PLoS Negl Trop Dis. 2014;8(5):e2876. doi: 10.1371/journal.pntd.0002876

30. Fayer R, Esposito DH, Dubey JP. Human infections with Sar cocystis species. Clin Microbiol Rev. 2015;28(2):295-311.

31. Lindsay DS, Dubey JP. Determination of the activity of pyrimethamine, trimethoprim, sulfonamides, and combinations of pyrimethamine and sulfonamides against Sarcocystis neurona in cell cultures. Vet Parasitol. 1999;82(3):205-210.
32. Shapiro K, Miller M, Mazet J. Temporal association between landbased runoff events and California sea otter (Enhydra lutris nereis) protozoal mortalities. J Wildl Dis. 2012;48(2):394-404.

33. Dubey JP, Zarnke R, Thomas NJ, et al. Toxoplasma gondii, Neospora caninum, Sarcocystis neurona, and Sarcocystis canis-like infections in marine mammals. Vet Parasitol. 2003;116(4):275-296.

34. McKenna PB, Charleston WAG. The survival of Sarcocystis gigantea sporocysts following exposure to various chemical and physical agents. Vet Parasitol. 1992;45(1-2):1-16.

35. Dubey JP, Saville WJ, Sreekumar C, et al. Effects of high temperature and disinfectants on the viability of Sarcocystis neurona sporocysts. J Parasitol. 2002;88(6):1252-1254.

36. Lindsay DS, Collins MV, Mitchell SM, et al. Survival of Toxoplasma gondii oocysts in Eastern oysters (Crassostrea virginica). J Parasitol. 2004;90(5):1054-1057.

37. Gibson AK, Raverty S, Lambourn DM, Huggins J, Magargal SL, Grigg ME. Polyparasitism is associated with increased disease severity in Toxoplasma gondii-infected marine sentinel species. PLoS Negl Trop Dis. 2011;5(5):e1142. doi: 10.1371/ journal.pntd.0001142

38. McClatchie S, Field J, Thompson AR, et al. Food limitation of sea lion pups and the decline of forage off central and southern California. $R$ Soc Open Sci. 2016;3(3):150628. doi: $10.1098 /$ rsos. 150628

39. Banuet-Martínez M, Espinosa-de Aquino W, ElorriagaVerplancken FR, et al. Climatic anomaly affects the immune competence of California sea lions. PLoS One. 2017;12(6):e0179359. doi: 10.1371/journal.pone.0179359

40. Bond NA, Cronin MF, Freeland H, Mantua N. Causes and impacts of the 2014 warm anomaly in the NE Pacific. Geophys Res Lett. 2015;42(9):3414-3420. 